\title{
Effect of soil resistivity on magnetic field in the case of lightning strike to a tall structure
}

\begin{abstract}
When a tower is struck by lightning, an induced voltage can generate in conducting objects within its vicinity. This phenomenon is due to the interaction between the electromagnetic field of the lightning with the object. The magnetic field is part of this field. Previous studies have shown that the magnetic field is affected by ground reflection coefficient factors that are usually taken as constants. However, the ground reflection coefficient factors have correlation with tower and ground impedance whereby the ground impedance can be determined based on soil resistivity and grounding system. This paper investigates the effect of soil resistivity on the value of magnetic field for a case of lightning strike on tall structures. Lightning current has been modelled accordingly and a set of equations is proposed to evaluate the magnetic field. Also, the effects of soil resistivity on the value of ground reflection coefficient factor consequently of the magnetic peak have been investigated. The results indicate that the magnetic field changes substantially with a variation of the soil resistivity. The magnetic field peak also has a strong correlation with the changes of soil resistivity as well as the fast front time of the channel base current. The outcome of the result of this paper will be beneficial to utility companies in determining the appropriate level of lightning protection since the magnetic field will be a result of induced voltage when have interaction with power lines.
\end{abstract}

Keyword: Component; Ground reflection factor; Lightning; Magnetic field; Soil resistivity; Tower 